\title{
Road Rage: Where to Go?
}

\author{
Anish Khadka ${ }^{1}$ and Inhi Kim ${ }^{2 *}$ \\ ${ }^{1}$ Southeast University-Monash University Joint Graduate School, China \\ ${ }^{2}$ Department of Civil Engineering Monash University, Australia
}

*Corresponding author: Inhi Kim, Department of Civil Engineering Monash University, Australia; Tel: Tel: +61- 3-9905-4240; Email: inhi.kim@monash.edu

Submission: 眥 July 25, 2018; Published: 眥 August 17, 2018

Keywords: Novaco anger scale; Sociodemographic; Probit model

Abbriviations: PADS: Angry Driving Scale; DAS Driving Anger Scale DBQ: Driving Behavior Questionnaire; DVQ: Driving Vengeance Questionnaire; NHTSA: National Highway Traffic Safety Administration; TAS: Trait Anger Scale

\section{Introduction}

Road rage is a deeply rooted social problem and has become a common phenomenon among road users. Over $85 \%$ of New South Wales (NSW) drivers interviewed reported of being a victim of various forms of road rage while this figure is even higher in Brisbane with 95\% [1]. Nine out of ten US drivers believe aggressive drivers pose a threat to their personal safety while $78 \%$ of the drivers report of engaging in aggressive driving at least once in 2014 AAAFTS [2]. The fact that road rage and aggressive driving is rated above drink driving as the biggest safety concern reveals the seriousness of the issue. Road rage can be defined as any hostile act conducted by a road user towards another road user on the roadway, including parking lots. These acts involve extreme forms of aggression like physically assaulting road users (both driver/passenger), vehicles or both. Road rage has become an easy medium to express anger, frustration, and the annoyance of everyday conflicts and disagreements on situations arising both on and off the roadway due to power, ease of escape, anonymity, and protection associated with vehicles.

Aggressive driving refers to reckless or dangerous driving in the absence of anger [3]. There is a thin line between aggressive driving and road rage with the only difference being the intention of the driver. Aggressive driving often results in discomfort to other road users and vice-versa; acting as a stimulus for either driver to engage in milder forms of aggression that escalates to road rage. Some of the milder forms of aggressive driving behavior include honking, cutting across lanes, tailgating, shouting/cursing, etc. The perpetrators of obscene gestures are also found to be equally on the receiving end of it. Hemenway et al. [4] reported that, of the $46 \%$ of drivers making obscene gestures to other drivers, $42 \%$ of them were found to be on the recipient end. These acts of aggressive driving and provocations reduce the road safety of both perpetrators as well as victims. Among the different aggressive driving behavior, speeding is considered as the most dangerous by NHTSA (National Highway Traffic Safety Administration). It is stated that $1 \mathrm{~km} / \mathrm{h}$ increase in mean traffic speed is associated with $3 \%$ increase in the incidence of injury crashes [5]. It is reported that more than $\$ 250.8$ million in hospital charges was incurred by aggressive driving-related inpatients in Ohio, 2004-2009 [6]. Smart \& Mann [7] found that road rage is often concealed as the cause of the accident with evidence from Ontario (1998), where $57.8 \%$ of fatal accidents involved unknown or reckless driving practices.

In the past literature, road rage/ anger has been measured by examining self-reported scales on how drivers express their anger under different driving scenarios using different measures like Propensity for Angry Driving Scale (PADS), Driving Anger Scale (DAS), Driving Behavior Questionnaire (DBQ), Driving Vengeance Questionnaire (DVQ), etc. As the construct definitions differ in each scale; these measures are heterogeneous [8]. Further, the factor structure of these questionnaires are found to vary across countries due to the cultural, environmental differences and also due to different analytical methods used; researchers have grouped DAS items into three factors in study conducted in the UK [9], six factors in the USA and Spain and five factors in France [9-12]. Leal \& Pachana [13] assessed the validity of Aus-PADS (Australian PADS) using various scales like novaco anger scale, impulsivity score, and venturesomeness score along with aggressive behaviors like yelling, gesturing at others. Deffenbacher et al. [10] state anger while driving as a situation-specific form of anger as it is influenced by many situational factors while the intensity of anger depends on both state and trait anger characteristics. As road rage and aggressive behavior are closely related, driving anger expression questionnaire like DAX (Driving Anger Expression) along with sociode- 
mographic characteristics like gender, income, age, marital status, education, etc., driving history like driving exposure (miles driven) and crash-related factors are used in the road rage studies. Wells Parker et al. [14] used driving behavior measure developed by James \& Nahl [15] with various items grouped together into three main subscales; verbal/frustration expression, threatening/angry driving, and direct contact/confrontation.

It is not surprising that most studies have found males, particularly young to be more likely associated with aggressive driving and traffic violations as compared to females due to their endorsement of reckless and angry driving style with motivating factors like sense of control, impression management, and thrill González Iglesias et al. [16]; Sullman et al. [17]. Wells Parker et al. [14] reported that road rage subscale score of males was twice as high as female while young drivers (age<30yrs) scored 3.5 times higher than old drivers (age $>50 y r s$ ). Researchers have contrasting view on the risk of aggression among females; Lonczak et al. [3] advocate that as the likelihood of driving with children is higher among females, it might lead to frustrations and distractions while driving and Rabasca [18] suggest females might release their stress at home on the roads by driving aggressively. It is evident that males and females differ significantly in both physical and psychological aspects. Thus, some of the driving situations that trigger aggressive driving behavior and the intensity of anger experienced among males might not have the same influence on females and vice-versa. Police presence is found to trigger anger among males while female's anger is associated with traffic obstruction $[3,16,17]$. Further, gender difference is also found in how drivers express anger; males adopt overt aggressive behavior like physical aggression while females adopt covert aggressive behavior like verbal aggression [19].

Wickens et al. [20] studied the potential of gender as moderator in understanding aggressive driving behaviors and accident risk factors. Most of the predictive factors like demographic characteristics and general risk factors were found to be consistent across genders. The difference in impact of education and income across gender is reported; risk of aggression is higher among females with university degree while males with post-secondary education. In case of salary, females with high-income level $(\$ 30,000-\$ 49,999)$ whereas males with medium income level (income $<\$ 30,000$ ) were found to be associated with higher risk of aggression. Regardless of gender, aggression is found to be common among drivers engaged in substance abuse like drink-driving, cannabis use, etc. and suffering from depression anxiety. Particularly among females, a strong positive association was found between driving anger and drinking frequency whereas negative associated between driving anger and drinking quantity [3]. DAS score was found to be unrelated to frequency of driving or exposure [21]. The presence of passenger influences the driving style, behavior, and degree of anger experienced for most drivers. However, the nature of the influence is bound to vary; the presence of family members or aged people generally has a calming effect while the presence of the passenger of similar age promotes rash/aggressive driving [22-24].

The modern-day living is characterized by fast-paced lifestyle and difficulties in attaining work-life balance. The stress, frustra- tion, anxiety, and annoyance of such lifestyle is fuelled by the delays resulting from traffic congestion; which more often increases the likelihood of aggression that is reflected in the driving behavior. Congestion was considered as the main cause of increasing road rage by about $86 \%$ of NSW drivers. Wickens et al. [20] found aggression to be prevalent among drivers reporting of stressful driving. Violations, like running red lights and honking abruptly, were found to be associated with frustrations resulting from congestion and delays [22]. Further, weather conditions associated with high temperature is found to induce anger with drivers reacting aggressively to stopped vehicles at the green light [25]. The horn latency, duration, and frequency can be used to assess aggression in such scenarios [26]. Shinar \& Compton [24] conducted an observational study and found that the frequency of aggressive behavior showed a strong linear relationship with congestion. However, further studying the relative risk of aggressive driving on delays associated with weekend and weekday congestion, it was found to be insignificant, highlighting that value of time is a determinant of aggressive behavior rather than congestion. This finding is also supported by Underwood et al. [27] Who did not find evidence of more anger experienced by the driver under high levels of congestion.

Road rage is found to be more common in urban areas as compared to rural areas [4]. The odds of experiencing different forms of road rage (milder or severe) is found to differ by regions [28]. Conner \& Smith [6] reported that greater proportion of aggressive driving in rural areas involved unsafe speed and following too closely in urban areas. Pfeiffer et al. [29] carried out a study among road rage victims at medico-legal center in Hamburg and reported that $68.1 \%$ of cases involved physical violence while in more than half of the cases involving objects utilized (31\%) used vehicle as a weapon. Positive correlation is found between road rage incidents and use of high-performance vehicles [30]. Kaysi \& Abbany [31] developed a binary probit model to predict aggressive drivers at un-signalized intersections using factors like sports car ownership, young age, and speed of vehicles on the major street. The negative impact of gun culture and its sensitivity towards road rage is evident from studies reporting positive correlations between the presence of a gun in vehicle and aggressive or illegal driving behavior [4,32]. Drivers with drink-driving behaviors, past records of accidents and tickets, and excessive speeding habit were found to be associated with threatening/angry driving subscale. Benavidez et al. [33] conducted a study on drug-dependent patients and found that the percentage of patients involved in road rage (perpetrator or victim) was significantly high as compared to general drivers as well as drivers reporting drug use in the past year. Serious forms of road rage like physical assault was found to be frequent among cocaine and both cocaine and heroin-dependent patients.

Underwood et al. [27] carried out a study by considering both questionnaires and diary approach to account for the fact that many accidents and details are forgotten by the respondents. Further, the author states that when considering crash involvement in the study; near crashes are more advantageous than actual crashes as their frequencies are comparable to the frequency of aggression experienced by drivers and they are also reported to have a signifi- 
cant association. This finding is also supported by the study carried out by Sullman et al. [17]. Most near crash scenarios were found to be followed by anger, especially when the driver is not at fault. The author states that low traffic density may provide more opportunities for rash drivers to drive carefree as compared to high traffic density, thus, increasing the chances of near accident and with it the potentially aggressive situations as well. Further, hostile actions and mild social deviance were found to be associated with both anger and near-accident experience. This highlights how aggressive and reckless driving behavior of other drivers can perpetuate road rage among victims and cause a ripple effect. The form of retaliation to offender's reckless driving; direct (victim engaging in aggressive driving behavior) or indirect (use laws like reporting to police to punish offender) is found to depend on the level of seriousness of the offense as well as trust and confidence in justice system [24].

With the ever-increasing auto ownership, the traffic demand exceeds infrastructural supply. Parking facilities, especially in urban areas are limited and disputes over reserved parking spaces of ten escalate to verbal and even physical aggression among road users. Drivers driving with high beam lights on at night, especially in highways lacking dividers separating opposing traffic movements is a common act of discourtesy that provokes anger among drivers. Among the anger-provoking driving situations in DAS, discourtesy is consistently rated as the highest in studies carried out among drivers in UK, USA, New Zealand, Tukey, France, and Malaysia [17]. The likelihood of aggression over discourtesy was found to be significant among novice and young drivers. Abdu et al. [35] conducted a study using driving simulator to analyze the causal relationship between driving ability and state anger. The participants were examined on both angry and normal state to analyze the difference in driving behavior under different states. State anger was found to increase risk-taking behaviors like exceeding speed limit instructed and crossing yellow lights; however, it did not compromise skill of the drivers (assessed by emergency maneuvers).

Apart from state anger, trait anger is a crucial aspect that governs the individual's propensity to become angry as well as the intensity of anger. This characteristic differs among individuals as some drivers have greater tendency to act aggressively towards provocations and external situational factors of both minor as well as major nature. TAS (Trait Anger Scale) is used to measure the trait anger. It is found to be associated with frequent involvement in crashes, aggressive driving, and anger towards other road users [36]. Deffenbacher et al. [21] did not find the correlation between trait anger and crash rates in their study, however, it was found to be associated with various crash risk conditions like loss of concentration, vehicular control, and close calls. Smoking is found to be associated with greater crash risk due to distractions [3].

Road rage is a deeply rooted problem as it can be associated with adolescence behaviors \& upbringing to various situational factors. Taubman Ben Ari \& Katz Ben Ami [37] examined the concept of "family climate for road safety" stating the importance of parental monitoring, guidance, control, communication and parent-adolescent relationship in shaping the adolescent behavior as it was found to be significantly associated with reckless driving and lack of commitment towards safety. Vassallo et al. [38] carried out a longitudinal study to address the claim that "antecedents of risky driving can be noticed at early ages prior to driving commencement". The author grouped drivers based on levels of risky driving behavior as low, medium, and high to analyze behavioral differences at various stages of child's development. Insignificant and minor differences were found between drivers belonging to different categories during infancy \& early childhood and childhood (mid and late) respectively. However, the differences were evident among low and high groups during early childhood (12-14 years) while the groups differed significantly during early adulthood (19-20 years). It is reported that the odds of self-reported driving aggression increase by two folds for individuals with conduct disorder (CD) as compared to $\mathrm{CD}$ absentees. Although, negative association was found between childhood CD and driver aggression, it wasn't significant [39].

The complex interaction between different modes and driver/ passenger behaviors are bound to result in frequent frustration and annoyance among road users. Deffenbacher et al. [40] studied the impacts of relaxation and cognitive-relaxation intervention for driving anger against no treatment case and concluded that both treatments lowered trait anger while increasing adaptive and constructive expression. It is essential to analyze both causes (state and trait) and consequences of aggressive driving behaviors and anger experienced by drivers to design interventions and strategies to reduce the increasing road rage.

\section{References}

1. GIO (2011) NSW drivers- still angry but slightly less so, compared to Brisbane.

2. Laura BD (2016) Prevalence of Self-Reported Aggressive Driving Behavior: United States, 2014. AAA Foundation for Traffic Safety, Washington, DC, USA.

3. Lonczak HS, Neighbors C, Donovan DM (2007) Predicting risky and angry driving as a function of gender. Accident Analysis \& Prevention 39(3): 536-545.

4. Hemenway D, Vriniotis M, Miller M (2006) Is an armed society a polite society? Guns and road rage. Accident Analysis \& Prevention 38: 687695.

5. Peden M, Scurfield R, Sleet D, Mohan D, Hyder AA, et al. (2004) World report on road traffic injury prevention. World Health Organization Geneva, Switzerland, pp. 1-244.

6. Conner KA, Smith GA (2014) The impact of aggressive driving-related injuries in Ohio, 2004-2009. Journal of safety research, 51: 23-31.

7. Smart RG, Mann RE (2002) Deaths and injuries from road rage: cases in Canadian newspapers. Canadian Medical Association Journal 167(7): 761-762.

8. Nesbit SM, Conger JC, Conger AJ (2007) A quantitative review of the relationship between anger and aggressive driving. Aggression and Violent Behavior, 12, 156-176.

9. Lajunen, T, Parker D, Stradling SG (1998) Dimensions of driver anger, aggressive and highway code violations and their mediation by safety orientation in UK drivers. Transportation Research Part F: Traffic Psychology and Behaviour 1(2): 107-121.

10. Deffenbacher JL, Oetting ER, Lynch RS (1994) Development of a driving anger scale. Psychological reports 74: 83-91. 
11. Sullman MJ, Gras ME, Cunill M, Planes M, Font Mayolas S (2007) Driving anger in Spain. Personality and Individual Differences 42(4): 701-713.

12. Villieux A, Delhomme P (2007) Driving anger scale, French adaptation: further evidence of reliability and validity. Percept Mot Skills 104(3): 947-957.

13. Leal NL, Pachana NA (2009) Validation of the Australian propensity for angry driving scale (aus-pads). Accid Anal Prev 41(5): 1112-1117.

14. Wells Parker E, Ceminsky J, Hallberg V, Snow RW, Dunaway G, et al (2002) An exploratory study of the relationship between road rage and crash experience in a representative sample of us drivers. Accid Anal Prev 34(3): 271-278.

15. James DL, Nahl D (2000) Aggressive driving is emotionally impaired driving. University of Hawaii, USA.

16. González Iglesias B, Gómez Fraguela JA, Luengo Martín MÁ (2012) Driving anger and traffic violations: Gender differences. Transportation Research Part F: Traffic Psychology and Behaviour 15(4): 404-412.

17. Sullman MJ, Stephens AN, Yong M (2014) Driving anger in Malaysia. Accident Analysis \& Prevention, 71: 1-9.

18. Rabasca L (1999) Happiness may increase with age. American Psychological Association Monitor, p.30.

19. Turner CW, Layton JF, Simons LS (1975) Naturalistic studies of aggressive behavior: aggressive stimuli, victim visibility, and horn honking. J Pers Soc Psychol 31(6): 1098-1107.

20. Wickens CM, Mann RE, Stoduto G, Butters JE, Ialomiteanu A, et al. (2012) Does gender moderate the relationship between driver aggression and its risk factors? Accid Anal Prev 45: 10-18.

21. Deffenbacher JL, Lynch RS, Oetting ER, Yingling DA (2001) Driving anger: Correlates and a test of state-trait theory. Personality and Individual Differences 31(8): 1321-1331.

22. Shinar D (1998) Aggressive driving: the contribution of the drivers and the situation. Transportation Research Part F: Traffic Psychology and Behaviour 1(2): 137-160.

23. Baxter JS, Macrae C, Manstead AS, Stradling SG (1990) Attributional biases and driver behaviour. Social behaviour.

24. Shinar D, Compton R (2004) Aggressive driving: an observational study of driver, vehicle, and situational variables. Accid Anal Prev 36(3): 429437.

25. Kenrick DT, Macfarlane SW (1986) Ambient temperature and horn honking: A field study of the heat/aggression relationship. Environment and behavior 18(2): 179-191.
26. Galovski TE, Blanchard EB (2004) Road rage: a domain for psychological intervention? Aggression and Violent Behavior 9(2): 105-127.

27. Underwood G, Chapman P, Wright S, Crundall D (1999) Anger while driving. Transportation Research Part F: Traffic Psychology and Behaviour 2(1): 55-68.

28. Smart RG, Stoduto G, Adlaf EM, Mann RE, Sharpley JM (2007) Road rage victimization among adolescents. J Adolesc Health 41(3): 277-282.

29. Pfeiffer JL, Pueschel K, Seifert D (2016) Interpersonal violence in road rage. Cases from the medico-legal center for victims of violence in Hamburg. Journal of Forensic and Legal Medicine 39: 42-45

30. Smart RG, Stoduto G, Mann RE, Adlaf EM (2004) Road rage experience and behavior: vehicle, exposure, and driver factors. Traffic Inj Prev 5(4): 343-348.

31. Kaysi IA, Abbany AS (2007) Modeling aggressive driver behavior at unsignalized intersections. Accid Anal Prev 39(4): 671-678.

32. Miller M, Azrael D, Hemenway D, Solop FI (2002) 'Road rage'in Arizona: armed and dangerous. Accid Anal Prev 34(6): 807-814.

33. Benavidez DC, Flores AM, Fierro I, Álvarez FJ (2013) Road rage among drug dependent patients. Accident Analysis \& Prevention 50: 848-853.

34. Dukes RL, Clayton SL, Jenkins LT, Miller TL, Rodgers SE (2001) Effects of aggressive driving and driver characteristics on road rage. The Social Science Journal 38(2): 323-331.

35. Abdu R, Shinar D, Meiran N (2012) Situational (state) anger and driving. Transportation research part F: Traffic Psychology and Behaviour 15(5): 575-580.

36. Dahlen ER, Martin RC, Ragan K, Kuhlman MM (2004) Boredom proneness in anger and aggression: effects of impulsiveness and sensation seeking. Personality and Individual Differences 37(8): 1615-1627.

37. Taubman Ben Ari O, Katz Ben Ami L (2013) Family climate for road safety: A new concept and measure. Accid Anal Prev 54: 1-14.

38. Vassallo S, Smart D, Sanson A, Harrison W, Harris A, et al. (2007) Risky driving among young Australian drivers: Trends, precursors and correlates. Accid Anal Prev 39(3): 444-458.

39. Wickens CM, Vingilis E, Mann RE, Erickson P, Toplak ME, et al. (2015) The impact of childhood symptoms of conduct disorder on driver aggression in adulthood. Accid Anal Prev 78: 87-93.

40. Deffenbacher JL, Filetti LB, Lynch RS, Dahlen ER, Oetting ER (2002) Cognitive-behavioral treatment of high anger drivers. Behav Res Ther 40(8): 895-910.
Creative Commons Attribution 4.0

International License

For possible submissions Click Here

\section{Submit Article}

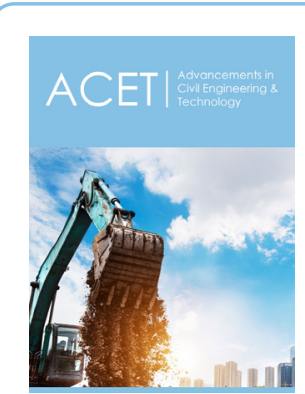

Advancements in Civil Engineering \& Technology

Benefits of Publishing with us

- High-level peer review and editorial services

- Freely accessible online immediately upon publication

- Authors retain the copyright to their work

- Licensing it under a Creative Commons license

- Visibility through different online platforms 